\title{
Estudio retrospectivo de las denuncias por malpraxis* médica en la provincia de Málaga.
} Retrospective study of medical malpractice suits in the province of Malaga (Spain).

\author{
F. Álvarez González' ${ }^{1}$ E. Hidalgo Salvador ${ }^{1}$, D. Sánchez del Castillo ${ }^{1}$ y \\ H. García Pérez ${ }^{1}$
}

\begin{abstract}
RESUMEN
Las denuncias contra los médicos son cada vez más frecuentes. Un hecho que hasta hace poco era relativamente anecdótico, se ha convertido en una situación jurídica habitual en la actualidad. El propósito de este estudio es recopilar los datos que desde nuestro punto de vista son más relevantes, de los existentes en los archivos del IML de Málaga, correspondientes a las denuncias por malpraxis en esta provincia. El objetivo es realizar una aproximación global, dada la nueva estructura del trabajo medico legal, a determinados aspectos periciales $y$ aportar nuevos criterios a la hora de realizar dichas periciales. Al mismo tiempo se analiza la práctica de la medicina en esta provincia y su relación con los pacientes.
\end{abstract}

Palabras clave: Malpraxis, responsabilidad médica, informe médico-forense.

Cuad Med Forense 2007; 13(47):9-19

\begin{abstract}
Suits against doctors are now more frequent than ever. A situation that was almost anecdotic some years ago have become very common nowadays. The aim of this study is to compile main data which, from our point of view, are more relevant of the obtained from the files of the Institute of Legal Medicine of Malaga (Spain) related to the medical malpractice suits in this province. The objective is to carry out an approach, taken into account the new structure of the medico-legal work, to some expert witness report and to propose new criteria to perform those evaluations. At the same time, the practice of medicine in this province and its relation with the patients are analyzed.
\end{abstract}

Key words: Malpractice, medical responsibility, forensic report.

* En los países anglosajones, las cuestiones relacionadas con la responsabilidad profesional médica se conocen con el termino "mal-practice", de donde ha surgido el neologismo "malpraxis", con el que se refieren a este problema algunas publicaciones actuales y cada vez más en la practica jurídica. No obstante, puesto que la RAE no reconoce aun esta palabra, puede sustituirse por "responsabilidad profesional médica"

Correspondencia: Dr. Eduardo Hidalgo Salvador. Instituto de Medicina Legal de Málaga, Servicio de Clínica, Palacio de Miramar, Paseo de Redding s/n, entreplanta. Telf.: 951035443, fax: 951035444. E-mail: eduardo.hidalgo.ius@juntadeandalucia.es

1 Médico Forense. Instituto de Medicina Legal de Málaga. 


\section{INTRODUCCIÓN:}

Las denuncias contra los médicos son cada vez más frecuentes. Un hecho que hasta hace poco era relativamente anecdótico, se ha convertido en una situación jurídica habitual en la actualidad. Este tema, que duda cabe, preocupa en gran manera al colectivo médico. Einstein dijo que la teoría es asesinada tarde o temprano por la experiencia. Sin duda, los profesionales médicos cuentan con un bagaje de conocimientos teóricos que han de contrastar diariamente mediante la práctica. Del mismo modo que no hay dos personas iguales, no existen dos pacientes que respondan de la misma forma a un tratamiento determinado. En el Instituto de Medicina Legal (IML) de Málaga, la realización de los Informes Médico Forenses sobre malpraxis médica supone un porcentaje inferior al $1 \%$ del total de informes periciales realizados anualmente. Estos informes se emiten por turno entre todos los Médicos Forenses del Servicio de Clínica excepto aquellos casos con resultado de muerte que son realizados por los Médicos Forenses del Servicio de Patología Forense.

Este tipo de Informe Médico Forense requiere indudablemente una especial dedicación de estudio y de creación intelectual, no existiendo un límite de tiempo para concluirlos dada la gran variabilidad existente, si bien debe estar dentro de un intervalo razonable.

Existen numerosos trabajos y revistas, fundamentalmente en el mundo anglosajón, dedicadas al estudio de la malpraxis médica. No olvidemos los altos costes de los seguros en estos países y las altas sumas de indemnización solicitadas por los pacientes, asuntos que progresivamente se van trasladando al resto de los países desarrollados. [I] Así encontramos estudios realizados en EE.UU. en los que se analiza el número de personas que sufre alguna lesión por negligencia en los hospitales. [2] En otro estudio llevado a cabo en California, se estimó que el 4,65 \% de la muestra de pacientes hospitalizados (20.864 pacientes en 23 hospitales) sufría algún percance, del que el 17\% se debía a negligencia médica. Otro estudio similar llevado a cabo en Nueva York, aseguraba que un 3,7\% de los pacientes de la muestra (31.429 pacientes en 51 hospitales seleccionados aleatoriamente) sufría algún tipo de daño, de los cuales el $28 \%$ era atribuible a negligencia médica. [3] En España J.J. Llovet [3] se sirvió de las memorias de la Organización Médica Colegial y, extrapolando los datos obtenidos de la Comunidad de Madrid, dedujo que el índice anual de reclamaciones por cada 100 facultativos era del 0,4\% en el año 1990. A pesar de esto, en España no hemos encontrado estudios referentes a este problema, si bien nos consta que las compañías de seguros médicos manejan sus propios estudios de mercado que no salen a la luz fácilmente.

Como se puede ver, los estudios realizados hasta ahora se centran en la problemática económica derivada de las denuncias por responsabilidad médica, tanto para el agraviado, los médicos implicados así como para los sistemas de salud pública y/o privados en los que se integran. El propósito de este estudio es recoger una serie de datos que, a nuestro criterio, son imprescindibles para entender la problemática que suscita este tema en el colectivo de profesionales sanitarios y de la que se pueden derivar implicaciones penales de los mismos.

\section{HIPÓTESIS DE TRABAJO:}

Las hipótesis que hemos tratado de comprobar han sido:

I. ¿Existen factores fuera de una actuación médica concreta que puedan influir en el inicio de la denuncia?.

2. iLas especialidades mas denunciadas son las de mayores requerimientos técnico-manuales o más comprometidas en sus resultados (por ejemplo, la Cirugía Plástica) o por el contrario se denuncian más los actos médicos genéricos?. 
3. ¿Cuál es el perfil medio del paciente que se ve inmerso en este tipo de denuncias en nuestro ámbito?

4. ¿Consta el consentimiento informado en todos los actos médicos denunciados como regula la ley 4I/2002? iEjerce dicho documento su papel protector del médico, así como del paciente?

5. ¿Cumple el ejercicio profesional en el ámbito privado un papel protector frente al sector público en las denuncias por malpraxis?

6. ¿Son adecuados los Informes Médico Forenses sobre malpraxis?. ¿Es necesario más de un Informe Médico Forense?; y sobre todo ¿Pueden introducir un factor de confianza en los profesionales médicos denunciados?

7. ¿Existe algún ámbito geográfico asistencial en nuestra provincia, en relación con su población, que se vea más involucrado en este tipo de actuaciones?

\section{MATERIAL Y MÉTODOS:}

Se han estudiado de forma retrospectiva el total de las denuncias por actos médicos que han sido estudiadas e informadas en el IML de Málaga desde su creación en septiembre de 2003, hasta el 30 de marzo de 2005 ( 19 meses), comprendiendo denuncias de hechos ocurridos desde 1997 hasta 2005.

El archivo se realiza por el personal de administración del IML, guardando copia de toda la documentación médica obrante en la causa, de las actuaciones judiciales relevantes, del informe médico-forense emitido así como una copia de la denuncia. A continuación se pasa la documentación al Jefe de Servicio de Clínica, el cual la turna a uno de los Médicos Forenses, siguiendo un orden establecido previamente, de manera que todos los Médicos Forenses integrados en este servicio realicen el mismo número de informes sobre malpraxis, independientemente del caso. Actualmente se ha modificado, adscribiendo dos Médicos Forenses a cada caso de responsabilidad profesional médica y llevando a sesión clínica aquellos casos cuyo estudio y resolución resultan más complejos.

Durante el período de estudio se han recogido 74 casos correspondientes a las denuncias por responsabilidad profesional médica tramitadas en el IML de Málaga desde su creación y centralización en el archivo de la Sede Central: 18 ocurrieron en el año 2003, 50 durante el año 2004 y 6 en el año 2005. La diferencia existente entre el año 2004 y los demás se debe a que no están recogidas todas los casos ya que el IML comenzó a funcionar en octubre de 2003 y la recogida de datos se hizo hasta principios de 2005, no incluyéndose todas la denuncias registradas en nuestro IML este año. Tampoco se han incluido en este estudio los casos con resultado de muerte o en las que se hubiera practicado autopsia judicial.

Se ha configurado una base de datos Excel en la que se han ido introduciendo los datos recogidos previamente de manera manual mediante la cumplimentacion de una hoja de datos. Los campos de estudio, seleccionados previamente a la recopilación de los datos, son los siguientes:

I. Estudio de demora suceso-denuncia y denuncia-informe

2. Inicio de la denuncia

3. Perjuicio ocasionado (muerte-lesiones)

4. Especialidad implicada

5. Falta médica

6. Tipo de informe pericial

7. Existencia de consentimiento informado 
8. Sexo y edad del perjudicado

9. Variable geográfica

10. Ámbito sanitario

Para dar mayor significación estadística a los datos recogidos, hemos desechados los valores extremos inferiores y superiores para evitar el falseamiento de los resultados. Por último, una vez recogidos los datos y plasmados en la base de datos, se ha procedido a realizar su procesamiento estadístico para extraer la información que a continuación se expondrá.

\section{RESULTADOS:}

I. ESTUDIO DE DEMORA SUCESO-DENUNCIA Y DENUNCIA-INFORME.

El plazo de tiempo entre la fecha de la actuación médica denunciada y la formalización de la denuncia en el juzgado alcanza los 528 días de media. La demora entre la fecha de recepción en el IML de Málaga de la documentación correspondiente a la denuncia por responsabilidad médica y la cumplimentacion del Informe Médico Forense alcanza los III días de media. La desviación estándar fue de 53,03 días.

\section{INICIO DE LA DENUNCIA.}

Se han considerado 5 posibilidades: Paciente, familiar, abogado, médico o de oficio (Gráfico n० I).

En 38 casos (51 \%), la denuncia la interpuso en propio perjudicado.

En 30 de los casos (40\%), la denuncia fue interpuesta por un familiar del perjudicado, generalmente el cónyuge y también, aunque menos frecuentemente, por descendientes.

En 8 de los casos estudiados (9\%) las denuncias fueron iniciadas por letrados en

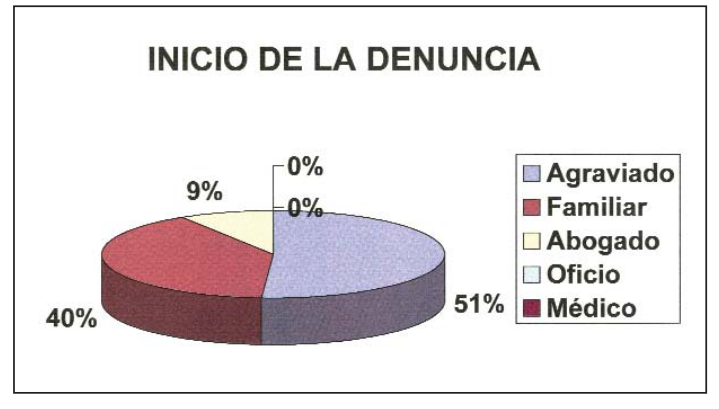

Gráfico 1 representación del perjudicado o de familiares del mismo.

En ninguno de los 74 casos estudiados las denuncias fueron iniciadas ni por facultativos ni de oficio.

\section{PERJUICIO OCASIONADO.}

Se ha dividido según el resultado del acto médico denunciado haya sido lesiones o muerte. El $66,6 \%$ de los actos médicos denunciados e incluidos en este estudio se debieron a lesiones (49 casos) y el 33,4\% restante se debió a actos médicos con resultado de muerte (25 casos). (Gráfico n 2 )

\section{ESPECIALIDADES MÉDICAS.}

En algunos casos, las denuncias por malpraxis afectan a varios profesionales por lo

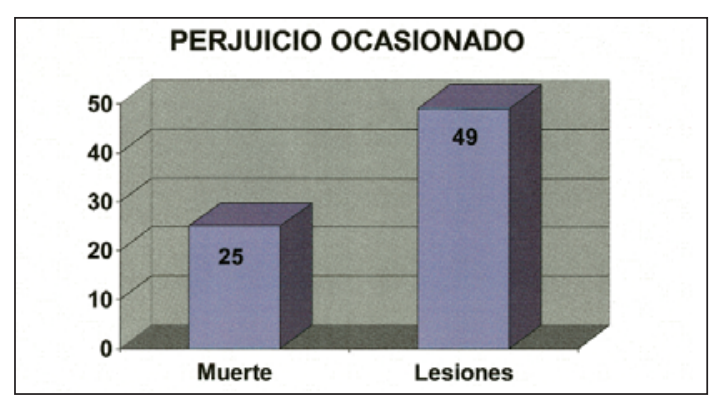

Gráfico 2 
que el número de casos sumados por especialidades implicadas es superior al número de casos estudiados. Obtenemos un total de 87 casos por especialidades implicadas. La distribución de los mismos es la siguiente:

- Traumatología y Cirugía ortopédica: I5 casos.

- Especialidades quirúrgicas: 13 casos.

- Asistencia primaria (incluyendo la "medicina de urgencias"): 12 casos.

- Ginecología y Obstetricia: II casos.

- Medicina Interna: 7 casos.

- Otorrinolaringología: 7 casos.

- Cirugía cardiovascular: 5 casos.

- Oftalmología: 4 casos.

- Psiquiatría: 3 casos.

- Pediatría: 3 casos.

- Urología: 2 casos.

- Anestesia: I caso.

- Cirugía plástica: 3 casos.

- Odontología: I caso.

Llama poderosamente la atención que las dos únicas especialidades que por definición deben garantizar el resultado esperado, Cirugía Plástica y Odontología (por contrato "de obra" y no "de medios" como el resto de las especialidades), sumen solo 4 denuncias entre ambas.

\section{FALTA MÉdICA.}

Se han dividido las denuncias realizadas entre aquellas en las que se pide que se estudie si existe malpraxis derivada de algún acto médico, de aquellas en las que de una manera más específica se solicita la emisión de informe en relación con algún hecho específico derivado de la asistencia médica. Nuevamente se dan más casos (93) que los realmente estudiados (74) puesto que en un mismo caso puede solicitarse el estudio de más de un aspecto derivado de la asistencia médica.

a) Los Informes Médico Forenses en los que hay que valoran el conjunto de las actuaciones médicas que se incluyen en la documentación que se aporta han supuesto el 75,2\% (70 casos) de los casos estudiados. Los Informes Médico Forenses en los que se especifica el hecho concreto sobre el que hay que valorar la existencia de responsabilidad profesional médica han supuesto el $24,8 \%$ restante (23 casos).

b) Entre las faltas médicas "concretas" hemos considerados las siguientes posibilidades:

- Asistencia despersonalizada: 7 casos

- Información insuficiente al paciente: 6 casos

- Falta de medios diagnósticos: 4 casos

- Falta médica en relación con la Historia Clínica: 3 casos

- Falta de cumplimiento de protocolos: 2 casos

- Omisión del deber de socorro: I caso

\section{Tipo de INFORMES PeRICIALES.}

Se han recogido todos los Informes Médicos periciales presentes en los casos estudiados, encontrándose tanto Informes Médico-Forenses como Informes Periciales Privados. 
Se ha emitido Informe Médico Forense en el $96 \%$ de los asuntos estudiados. En el $4 \%$ de los casos no se emitió Informe Médico Forense porque se archivaron las diligencias antes de poder emitir nuestra pericial. Solo en un 15\% de los casos estudiados se ha encontrado Informe Médico Pericial Privado.

Los Informes Médico Forenses desestimaron la existencia de malpraxis médica en un $82,5 \%$ de los casos mientras que en un 13,5\% de los casos, estimaron la existencia de la misma. (Gráfico n 3)

En los casos en los que constan Informes Periciales Privados, estos negaron la responsabilidad profesional médica en un

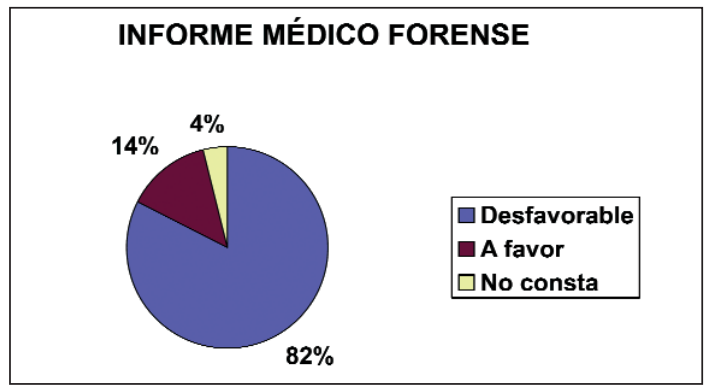

Gráfico 3 $72 \%$ de los casos. En un 28\% de los casos estimaron la existencia de malpraxis médica.

\section{CONSENTIMIENTO INFORMADO.}

Se ha estudiado la existencia del consentimiento informado en la documentación médica obrante en las diligencias como condición previa al acto médico denunciado. Se ha encontrado documento informando al paciente $y / 0$ a los familiares en un $15 \%$ de los casos, mientras que en un $54 \%$ de los casos no existía y en el $31 \%$ restante se ignora su cumplimentacion. (Gráfico n 4)

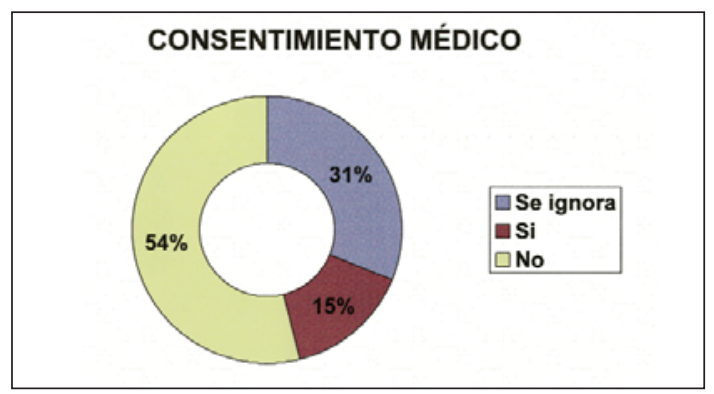

Gráfico 4

\section{SEXO Y EDAD DEL PERJUDICADO.}

La distribución de casos según el sexo de los afectados no encuentra grandes diferencias, encontrándose un mayor número de asuntos en los que los afectados eran mujeres (54\%).

Las cuestiones de responsabilidad profesional médica en que los afectados eran varones suponen un $46 \%$. (Gráfico n 5 )

En cuanto a la distribución por edades, el grupo de edad donde mayor número de afectados encontramos es el de los mayores de 60 años, siendo el comprendido entre 0 y 15 años, el grupo de edad menos denunciado. (Gráfico n 6)

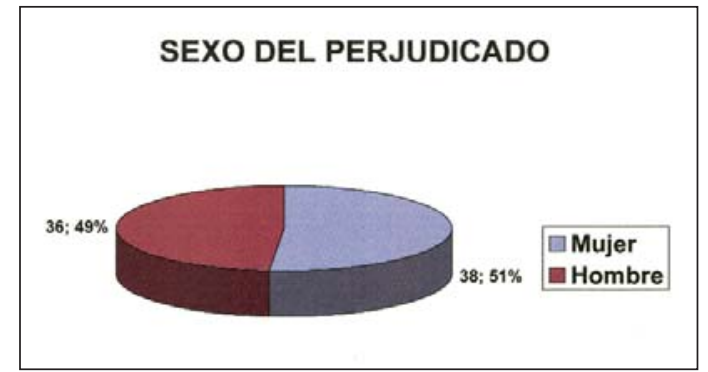

Gráfico 5

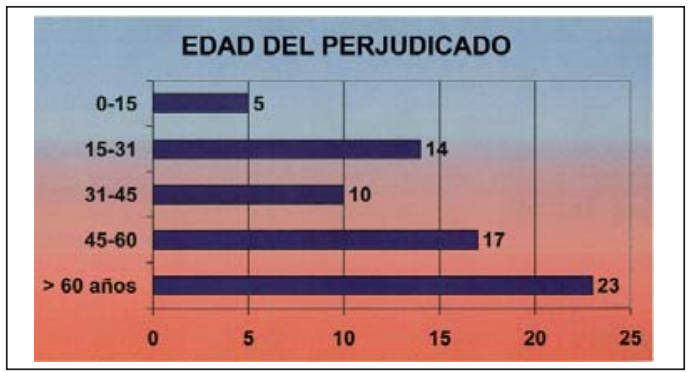

Gráfico 6 
Los datos referentes a la nacionalidad del denunciante en los casos acreditados se ha observado que más del $90 \%$ son españoles.

\section{VARIABLE GEOGRÁFICA.}

Hemos tratado de encuadrar las denuncias en varias zonas geográficas dentro de la provincia de Málaga. Así hemos subdividido la provincia de Málaga por sectores correspondientes al Juzgado que ha instruido la causa y la localización de los principales centros sanitarios de la provincia I. Sector Málaga; 2. Sector Costa del Sol (incluidos Marbella, Coín, Estepona, Ronda, Fuengirola y Torremolinos); 3. Sector Axarquia; 4. Sector Antequera-Archidona. (Gráfico $n^{\circ} 7$ )

Un 73\% de los asuntos por responsabilidad médica estudiados (55 casos) se han instruido en Málaga. En Juzgados de los incluidos en el Sector Costa del Sol se han instruido 13 casos ( 17\%) y de los otros 2 sectores, 4 casos en cada uno (5\%).

Otro dato interesante que hemos recogido ha sido la incidencia de denuncias por Centros Sanitarios (tanto públicos como privados), (encontrando que en algunos casos el Juzgado de la denuncia no ha sido el instructor). Los centros sanitarios de Málaga abarcan el grueso de las denuncias con 5 I casos denunciados, distribuidos de la siguiente manera: 23 casos se denunciaron por supuestas malpraxis ocurridas en centros sanitarios del "Distrito Centro de Málaga". En el "Sector Oeste de Málaga" se denunciaron 19 casos de malpraxis y en el "Sector Este de Málaga" 9 casos. El resto de las responsabilidades médicas estudiadas tuvieron su origen en los siguientes centros: 7 casos en la Axarquía; 4 casos en Antequera; 5 casos en Benalmadena; 2 casos en Marbella y otros 2 en Torremolinos.

\section{Asistencia Pública o Asistencia Privada.}

En 39 casos de los estudiados (52\%) la asistencia se realizó en el sistema sanitario público, en 33 casos (44\%) en el sistema sanitario privado y en 3 casos (4\%) se han visto implicados ambos sistemas. (Gráfico n 8)

\section{DISCUSIÓN:}

No se han podido comprobar de manera objetiva las causas que explican la gran demora existente entre la producción de los hechos objeto de la querella y la

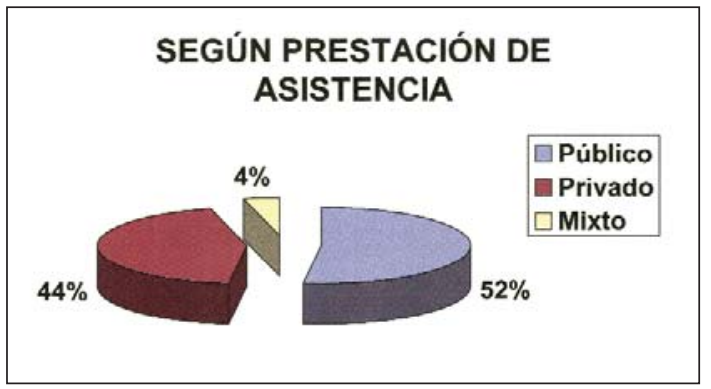

Gráfico 8 interposición de la misma, 528 días de media. Dado que para el inicio del estudio de una posible responsabilidad médica no es exigible que exista una motivación, es esta una cuestión que no queda reflejada en las diligencias practicadas. Sin embargo, los autores del estudio consideramos subjetivamente que en la actualidad no solo actúa como detonante el acto medico presuntamente negligente, sino otro tipo de circunstancias (segundas opiniones, informaciones 
generales, medios de prensa, intereses económicas, etc.), que no hemos podido constatar en los diversos expedientes por la razón anteriormente esgrimida.

A pesar de no poder probar objetivamente cual es la causa de la demora, del minucioso estudio de los historiales médicos que han sido base para este trabajo, se puede deducir, que en la mayor parte de los casos, incluidos los que tuvieron como resultado el fallecimiento del paciente, no se trata de patologías que, como media, tengan un periodo de curación/convalecencia muy prolongado. De hecho, las dos especialidades en las que más casos de responsabilidad médica hemos tenido que valorar, Traumatología/Cirugía Ortopédica y Asistencia Primaria (27 casos entre ambas), se ocupan de patologías que, en principio, cabe esperar que se resuelvan en un periodo no muy largo de tiempo.

El tiempo medio en la emisión de los informes Médico Forenses, I I I días desde la recepción de la documentación en este IML, se debe entre otros a la dificultad que supone, por lo general, el estudio pormenorizado de cada caso y a la necesidad de solicitar documentación imprescindible para su resolución. Pero es sin duda, el primer extremo comentado el que mayor dilatación en los periodos de entrega produce, ya que el estudio del caso, la actualización de los conocimientos científicos que en la mayoría de los expedientes es necesaria y la redacción de un informe comprensible para Magistrados, Jueces, Fiscales y Tribunales requiere una dedicación más prolongada que para otro tipo de Informes Forenses. Los informes sobre responsabilidad profesional médica, son una de las actividades científicas más completas y complejas para el Médico Forense.

Contrariamente a lo esperado por los autores de este estudio las dos únicas especialidades que por definición deben garantizar el resultado esperado (por contrato "de obra" y no "de medios" como el resto de las especialidades), sumen solo 4 denuncias entre ambas. En concreto, Cirugía Plástica y Reparadora ha supuesto solo 3 casos de malpraxis médica y la odontología ha supuesto un solo caso. Encontrándonos resultados más acordes con la implicación de otras especialidades como Traumatología o Ginecología-Obstetricia.

El consentimiento informado como condición previa a la realización de cualquier acto médico, solo aparece recogido o aportado a las diligencias en apenas el 13\% de los casos (a pesar de que es de obligada cumplimentacion desde el año 2002). Los autores consideran que cuando se cumplimenta debida y pormenorizadamente, supone una garantía especial y facilita la realización del informe pericial y sus conclusiones.

Otro aspecto muy interesante de este estudio ha sido comprobar que en la mayoría de los casos se solicita el estudio de la documentación para ver si del conjunto de las actuaciones médicas recogidas se deriva o no responsabilidad profesional médica. Es decir, no se denuncia un hecho concreto de la actuación médica ni se aportan pruebas o informes para apoyarlas, sino que se denuncia verbalmente el hecho percibido por el perjudicado sin más asesoramiento que la asistencia recibida en general. Se podría resumir como "denuncia a ver que pasa".

En cuanto a las conclusiones de los informes periciales obrantes en la documentación aportada, destaca que los Informes Médico Forenses han descartado la existencia de malpraxis médica derivada de los hechos denunciados en un $82,5 \%$ de los casos estudiados, mientras que los Informes Periciales Privados, generalmente a posteriori, excluyeron la existencia de responsabilidad médica en un $72 \%$ de los casos. Por lo tanto parece existir una correlación entre las conclusiones de los Informes Periciales Medico Forenses y Privados, a pesar de que el número de informes privados existentes en los casos estudiados es sensiblemente inferior al de Informes Médico Forenses. En el momento del estudio de la documentación médica obrante en la causa, no siempre consta Informe Pericial de Parte. Debemos suponer que o bien se aportará con posterioridad a nuestro Informe o bien no fue necesario. 
En contra del sentir general del colectivo médico, aun cuando el número de denuncias por presuntas negligencias médicas crece año tras año de forma considerable, el número de situaciones procesales que implican objetivamente una malpraxis en relación con el número de actos médicos, es todavía muy pequeño.

Respecto a las otras variables estudiadas, en el sexo parece haber una cierta igualdad como parece lógico pensar entre denuncias mujer-hombre, siendo solo algo más de la mitad las que afectan a mujeres.

Sobre la edad también parece adecuado que la mayoría de los implicados tenga más de 60 años. Como norma general, a mayor edad, mayor es la posibilidad de sufrir enfermedades y por tanto es más probable que requieran asistencia médica.

Respecto de la nacionalidad, consideramos algo extremo que más del $90 \%$ de los denunciantes sean españoles dada la gran colonia extranjera, de avanzada edad y residente en la provincia de Málaga. No podemos teorizar sobre algo que nos es completamente ajeno, pero quizás entre los motivos por los que obtenemos este dato en nuestro estudio se encuentren la falta de confianza, del extranjero, en el sistema judicial español o incluso por las dificultades que pueda introducir el idioma. De hecho, según se desprende de todos los medios, la conciencia de denuncia por malpraxis en otros países del ámbito europeo está muy extendida.

De los resultados obtenidos se aprecia un cierto equilibrio entre los casos de responsabilidad profesional médica en la asistencia médica de carácter público con respecto a la asistencia médica de carácter privado (incluyendo compañías médicas). Esta armonía, en opinión de los autores, parece contradecir el sentimiento generalizado dentro de la profesión médica de que el ejercicio privado protege frente a estas denuncias. Recordemos que, aunque sin datos estadísticos, la asistencia pública genera un mayor número de actuaciones médicas en comparación con el número de actos médicos realizados en el marco del sector privado.

Sobre la distribución geográfica, es Málaga capital y sus centros hospitalarios los que recogen mayor numero de denuncias, lo cual tiene clara explicación al ser referencia y asistir a una mayor población durante todo el año.

\section{CONCLUSIONES:}

En respuesta a las hipótesis planteadas, podemos establecer las siguientes conclusiones:

I. Los casos en los que hay que valoran el conjunto de las actuaciones médicas de forma global son abrumadoramente mayoritarias frente a los casos en los que se denuncia un aspecto concreto de la actuación médica. (y con escasa aportación técnica?). Destacan por especialidades en primer lugar Traumatología, seguida de Cirugía, Medicina General y Urgencias, así como Ginecología y Obstetricia, encontrado que las especialidades que deben garantizar el resultado, como Cirugía Plástica u Odontología, representan un porcentaje mínimo de las denuncias estudiadas.

2. En cuanto a la provincia de Málaga, el perfil del perjudicado sería el de una mujer, de más de 60 años de edad y de nacionalidad española.

3. El consentimiento informado debe ser una pieza fundamental en la relación médicopaciente y cuanto más amplio y explícito, más realidad y protección ofrecerá al médico como al paciente, siendo una pieza fundamental para la resolución de los casos de malpraxis. No obstante, de nuestros resultados se desprende que, hasta el momento, no ha tenido la relevancia esperada. 
4. No se aprecia ningún papel protector de la asistencia médica en el marco del sector privado sobre la pública, a la vista los resultados obtenidos.

5. En la inmensa mayoría de los casos, los Informes Médico Forenses no solo son adecuados para la resolución de estos casos sino además imprescindibles, bien porque son los únicos, o porque entran directamente en el fondo de la actuación médica motivo de estudio, sin dejar mucho margen para las interpretaciones. Además, dado el porcentaje de informes desfavorables, en alta coincidencia con otros informes periciales, debe introducir un alto nivel de confiabilidad tanto para los médicos como para los pacientes. En casi todos los casos el único Informe Medico-Forense fue suficiente para el sobreseimiento de las actuaciones o para la continuación de las actuaciones.

6. Los datos obtenidos parecen adecuados en relación a la asistencia que se presta en las distintas zonas geográficas de la provincia.

\section{RECOMENDACIONES MÉDICO-FORENSES [5]:}

- La emisión de informes no debería ser excesivamente larga y prolija, descartando los casos en los que no se cumplan los elementos constitutivos de responsabilidad médica.

- Deberían establecerse canales de consulta oficial con determinadas especialidades para aquellos casos de especial dificultad.

- Los casos de especial complejidad deberían ser discutidos y evaluados en sesión clínica, enviando previamente copia para el resto de forenses con objeto de facilitar su resolución.

- Establecer claramente los objetos de la pericia ciñéndose a lo solicitado por el juzgado y en espera de nuevas solicitudes.

- Solicitar y obtener toda la historia clínica, así como declaraciones y otros datos de interés.

- Dada su especial complejidad, las actuaciones en juicio oral deberían ser realizados por un Médico Forense ponente y otro que actuara conforme. En estos casos seria aconsejable fueran visados por el Jefe del Servicio de Clínica Médico Forense.

- No olvidar principios básicos de la malpraxis "EL ERROR DIAGNOSTICO (*) NO ES PUNIBLE, SOLO ES EXIGIBLE LA PRÁCTICA DE LAS MEDIDAS ELEMENTALES Y NECESARIAS PARA LA RESOLUCIÓN MÉDICA DE CADA CASO" [6-7].

\section{RECOMENDACIONES A LOS MÉDICOS EN EL EJERCICIO PROFESIONAL:}

- Realizar historias clínicas detalladas y completas.

- No manipular "NUNCA" dichas historias.

- Realizar siempre consentimientos informados protocolizados, detallados y bien cumplimentados. Si por alguna circunstancia, el consentimiento se obtiene verbalmente, debe ser en presencia de testigos lo más imparciales posibles.

${ }^{*}$ ()En principio, el error diagnostico carece de relevancia penal, pero ello salvo en los casos en que por su entidad y dimensiones, constituya una equivocación inexcusable, ya sea por conductas descuidadas de las que resulte un proceder irreflexivo, derive de la falta de adopción de cautelas de generalizado uso o de la ausencia de pruebas, investigaciones o verificaciones precisas como imprescindibles para seguir el curso en el estado del paciente. 
- No rehuir la entrevista medica a los familiares, sino intentar dar la explicación médica de los hechos.

- Declarar cuando sean requeridos con claridad y ciñéndose a temas médicos, no olvidando que sobre su materia cada uno puede ser un perito cualificado $[8,9]$.

- Exigir de los Colegios Oficiales de Médicos, Servicios Sanitarios Públicos y de Compañías de Seguros la adecuada cobertura profesional y pericial experta en estos temas y no en los genéricos.

- Intentar siempre conservar la calma en el trato con los pacientes.

- No realizar comentarios despectivos hacia otros facultativos en presencia del paciente a no ser que se sea capaz de demostrarlo posteriormente en los juzgados.

\section{BIBLIOGRAFÍA:}

I.- Danzón PM. The crisis in medical malpractice: a comparison of trends in the United States, Canada, the United Kingdom and Australia. Law Med Health Care 1990;18:48-58.

2.- Eddy DM. The use of evidence and cost efectiveness by the courts: how can it help improve health care? J Health Polit Policy Law 2001;26:387-409.

3.- Llovet J]. Las reclamaciones judiciales por mala praxis médica. Rev Esp Salud Pública 1993;67:315-23

4.- Medicina Legal y Toxicología, G. Calabuig y E. Villanueva, $6^{a}$ edición. 2004. Editorial Masson. "Características y peculiaridades del contrato médico", pag. 71
5.- Tzeel A. Clinical practice guidelines and medical malpractice. Physician Exec 2002 Mar-Apr;28(2):36-9.

6.- Havard J. D. The medical malpractice position in the United Kingdom.., Law Med Health Care 1986 Sep;14(3-4):175-9.

7.- Dirnhofer R, Wyler D. Treatment failure from the viewpoint of forensic medicine. Ther Umsch 1997 May;54(5):272-9.

8.- McCarter WD, Hayek TJ Expert medical testimony is generally required to prove medical negligence., Mo Med. 2001 $0 \mathrm{ct} ; 98(10): 488-9$.

9.- Hayek TJ. Exceptions to expert testimony requirement in a medical negligence case. Mo Med. 2002 Jan;99(I):17-8. 